\title{
MODULAR GROUP ALGEBRAS OF TOTALLY PROJECTIVE $p$-PRIMARY GROUPS
}

\author{
WARREN MAY
}

\begin{abstract}
Let $F$ be a field of characteristic $p>0$ and let $G$ be a totally projective abelian $p$-group of countable $p$-length. If $F G \cong F H$ for some group $H$, then it is shown that $G \cong H$.
\end{abstract}

Let $F$ be a field of characteristic $p>0$ and let $G$ be an (infinite) abelian p-group. The question of whether $F G \cong F H$ implies that $G \cong H$ has been considered by Berman and Mollov [1] and May [5]. (For the finite nonabelian case, see Passman [8].) It is shown in [1], [5] and by Dubois and Sehgal [2] that the Ulm-Kaplansky invariants of $G$ and $H$ must be equal. Thus one can easily settle the case when $G$ is countable. Since the totally projective $p$-groups form the largest natural class of abelian $p$-groups that are determined by their Ulm-Kaplansky invariants, one would like to know in general whether $H$ must be totally projective if $G$ is. In this direction, Berman and Mollov give an affirmative answer if $G$ is a direct sum of cylic groups. By utilizing a theorem of Hill [4], we shall extend these results to $p$-groups of countable $p$-length. Precisely, we shall prove:

THEOREM 1. Let $R$ be a commutative ring with identity in which $p$ is not invertible, and let $\Omega$ be the first uncountable ordinal. Let $G$ be an abelian p-group of p-length $<\Omega$ whose reduced part is totally projective. If $H$ is a group such that $R G$ and $R H$ are isomorphic algebras, then $H$ is isomorphic to $G$.

To deduce Theorem 1, we shall prove several facts about the group of units of $R G$. For any commutative ring $R$ with identity and group $G$, let $U(R G)$ denote the group of units of $R G$ that have augmentation 1 (i.e., the coefficients sum to 1 ). We prove:

THEOREM 2. Let $R$ be a commutative ring with identity of characteristic $p$ that is perfect (i.e., every element is a pth power). Let $G$ be an abelian p-group of $p$-length $\leqslant \Omega$ whose reduced part is totally projective. Then $U(R G)$ is an abelian p-group of the same p-length as $G$, and whose reduced part is totally projective. Moreover, $G$ is a direct factor of $U(R G)$.

We remark that Mollov [6], [7] has investigated the structure of unit groups under certain restrictions.

Received by the editors August 19, 1978.

AMS (MOS) subject classifications (1970). Primary 20K10; Secondary 16A26.

Key words and phrases. Totally projective $p$-groups, modular group algebras. 
Before proving two preliminary lemmas, we must briefly discuss $p$-heights. Groups will be written multiplicatively, thus we shall use $G^{p^{\sigma}}$ ( $\sigma$ an ordinal) to denote the subgroup of $G$ consisting of elements with $p$-heights $\geqslant \sigma$. Suppose that $R$ is a commutative ring with identity of characteristic $p$, and let $G$ be an abelian $p$-group. Then $U(R G)$ is a $p$-group and consists precisely of the elements of $R G$ of augmentation 1. Suppose further now that $R$ is perfect. Then $U(R G)^{p}=U\left(R\left(G^{p}\right)\right)$, thus it follows that $U(R G)^{p^{o}}=U\left(R\left(G^{p^{o}}\right)\right)$ for every ordinal $\sigma$. Let $\alpha \in U(R G)$ and consider the support of $\alpha$, i.e. those $g \in G$ that appear nontrivially in $\alpha$. Clearly the $p$-height of $\alpha$ in $U(R G)$ is the minimum of the $p$-heights in $G$ of the elements in the support of $\alpha$. In particular, if we regard $G$ as a subgroup of $U(R G)$, then it is an isotype subgroup. We also observe that the $p$-length of $U(R G)$ is clearly equal to the $p$-length of $G$.

LEMMA 1. Let $R$ be a perfect commutative ring with identity of characteristic $p$, and let $G$ be an abelian p-group.

(a) Let $A$ be a finite subgroup of $G$, and let $N$ be a subgroup of $U(R A)$. Then $N$ is a nice subgroup of $U(R G)$.

(b) If $G$ is countable, then the reduced part of $U(R G)$ is totally projective.

Proof. Let $\alpha \in U(R G)$ and let $S=\{g a \mid g \in$ (support $\alpha$ ), $a \in A\}$. If $\beta \in N$, then the $p$-height of $\alpha \beta$ equals the $p$-height of some element in the finite set $S$. Therefore $\beta$ can be chosen to maximize this $p$-height. Hence $N$ is nice in $U(R G)$.

Now suppose that $G$ is countable, and let $A_{1} \subseteq A_{2} \subseteq \cdots$ be finite subgroups of $G$ with $\cup_{i<\omega} A_{i}=G$. For each $i$, we can choose a family $\left\{N_{i \alpha}\right\}$ of subgroups of $U(R G)$ indexed by an initial segment of ordinals such that $U\left(R A_{i}\right) \subseteq N_{i \beta} \subseteq N_{i \alpha} \subseteq U\left(R A_{i+1}\right)$ if $\beta<\alpha, N_{i \alpha}=\cup_{\beta<\alpha} N_{i \beta}$ if $\alpha$ is a limit ordinal,

$$
N_{i 0}=U\left(R A_{i}\right), \quad \bigcup_{\alpha} N_{i \alpha}=U\left(R A_{i+1}\right), \quad \text { and } \quad\left(N_{i, \alpha+1}: N_{i \alpha}\right)=p .
$$

Each $N_{i \alpha}$ is nice in $U(R G)$ by (a), therefore $\cup_{i<\omega}\left\{N_{i \alpha}\right\}$ is a nice decomposition series for $U(R G)$. It follows that the reduced part of $U(R G)$ is totally projective.

LEMMA 2. Let $R$ be a commutative ring with identity and let $G$ be an abelian group. Suppose that there exists a group $B$ such that $G$ is isomorphic to a direct factor of $U(R B)$. Then $G$ is a direct factor of $U(R G)$.

Proof. Let $G$ be isomorphic to the direct factor $V$ of $U(R B)$. Regarding $V$ as a subgroup of $U(R B)$, the isomorphism $G \rightarrow V$ induces a ring homomorphism $R G \rightarrow R B$, and hence a homomorphism $U(R G) \rightarrow U(R B)$. By composing with a projection of $U(R B)$ onto $V$, we obtain a homomorphism $U(R G)$ $\rightarrow V$ that maps $G$ isomorphically to $V$. Thus $G$ is a direct factor of $U(R G)$.

Proof of Theorem 2. We have already observed that $U(R G)$ is an abelian 
$p$-group of the same $p$-length as $G$. We shall now show that the reduced part of $U(R G)$ is totally projective by using induction on $|G|$. The countable case is done by Lemma 1 , therefore we may suppose that $G$ is uncountable. The assumption that $G$ has totally projective reduced part of $p$-length $\leqslant \Omega$ means precisely that $G$ is isomorphic to a direct sum of countable $p$-groups (see [3, Theorem 82.4]). Let $\tau$ be the first ordinal with $|\tau|=|G|$. We may assume that $G=\amalg_{\beta<\tau} A_{\beta}$, where each $A_{\beta}$ is countable. For each $\alpha<\tau$, put $G_{\alpha}=\amalg_{\beta<\alpha} A_{\beta}$ and $U_{\alpha}=U\left(R G_{\alpha}\right)$. (Note that $G_{0}=1$.) The projection $G_{\alpha+1} \rightarrow G_{\alpha}$ with kernel $A_{\alpha}$ induces a surjective map $U_{\alpha+1} \rightarrow U_{\alpha}$ that is idempotent. Thus we obtain an inner direct splitting $U_{\alpha+1}=U_{\alpha} \times K_{\alpha}$. It is easily seen that $U(R G)=$ $\amalg_{\alpha<\tau} K_{\alpha}$. But $|\alpha+1|<|G|$, thus $\left|U_{\alpha+1}\right|<|G|$, and consequently the reduced part of $U_{\alpha+1}$ is totally projective by induction. It now follows that $U(R G)$ has totally projective reduced part.

To show that $G$ is a direct factor of $U(R G)$ let $B=\amalg_{i<\omega} G$. Then the reduced part of $U(R B)$ is totally projective by what we have just shown. Let $f_{\sigma}(G)$ denote the $\sigma$ th Ulm-Kaplansky invariant of $G$. We know that $f_{\sigma}(B) \leqslant$ $f_{\sigma}(U(R B))$ since $B$ is isotype in $U(R B)$, therefore $f_{\sigma}(U(R B))=f_{\sigma}(G)+$ $f_{\sigma}(U(R B))$ for every ordinal $\sigma$. Moreover, if $D_{G}$ and $D_{U}$ are the maximal divisible subgroups of $G$ and $U(R B)$, respectively, then $D_{U} \cong D_{G} \times D_{U}$. Hence, $U(R B) \cong G \times U(R B)$ since we are dealing with totally projective reduced parts. By Lemma 2, we conclude that $G$ is a direct factor of $U(R G)$.

Since the complement of $G$ in $U(R G)$ has totally projective reduced part, one could determine it up to isomorphism by computing the rank of its maximal divisible subgroup and its Ulm-Kaplansky invariants in terms of $R$ and the Ulm-Kaplansky invariants of $G$. We remark that this is a feasible computation, but we shall not discuss it here.

Proof of Theorem 1. Our assumptions on $R$ guarantee that there is a homomorphism (not necessarily surjective) $R \rightarrow F$, where $F$ is an algebraically closed field of characteristic $p$. It follows that $F G \cong F H$. One easily sees that $H$ must be an abelian $p$-group since a torsion-free element in $H$ would be transcendental over $F$, while an element of order relatively prime to $p$ would yield a nontrivial idempotent in $F H$. Moreover, the maximal divisible subgroups of $G$ and $H$ are isomorphic by reference to [5, Corollary 7].

We may assume that the isomorphism $F G \cong F H$ preserves augmentations, hence $U(F G) \cong U(F H)$. Theorem 2 allows us to conclude that the reduced part of $U(F H)$ is totally projective of countable $p$-length. Thus the reduced part of $H$ is isomorphic to an isotype subgroup of countable $p$-length in the reduced part of $U(F H)$. It now follows immediately from a result of Hill [4, Theorem 1] that the reduced part of $H$ is totally projective. As was mentioned in the introduction, the Ulm-Kaplansky invariants of $G$ and $H$ are known to be equal; therefore $G \cong H$.

We conclude with several questions and remarks. Does Theorem 1 hold if $G$ has $p$-length $\geqslant \Omega$ ? In this case, we no longer have Hill's theorem to apply. 
Even more, there is uncertainty whether Theorem 2 holds if the $p$-length is $>\Omega$ since $G$ would not be a direct sum of countable groups. If one tries to prove Theorem 2 by induction on the $p$-length of $G$, the difficult step seems to occur at isolated ordinals. As a final question, if $G$ is a torsion-complete abelian $p$-group, then is $H$ torsion-complete? If so, then one could conclude $G$ and $H$ would be isomorphic since the Ulm-Kaplansky invariants serve to classify torsion-complete groups.

\section{REFERENCES}

1. S. D. Berman and T. $\mathrm{Zh}$. Mollov, The group rings of abelian p-groups of arbitrary power (Russian), Mat. Zametki 6 (1969), 381-392; Math. Notes 6 (1969), 686-692. MR $40 \$ 7365$.

2. P. F. Dubois and S. K. Sehgal, Another proof of the invariance of Ulm's functions in commutative modular group rings, Math. J. Okayama Univ. 15 (1972), 137-139. MR 47 \$6892.

3. L. Fuchs, Infinite abelian groups, vol. II, Academic Press, New York, 1973.

4. P. Hill, Isotype subgroups of direct sums of countable groups, Illinois J. Math. 13 (1969), 281-290. MR $39 \sharp 1550$.

5. W. May, Commutative group algebras, Trans. Amer. Math. Soc. 136 (1969), 139-149. MR 38 $\# 2224$.

6. T. Zh. Mollov, The multiplicative groups of modular group algebras of primary abelian groups of arbitrary cardinality. I (Russian), Publ. Math. Debrecen 18 (1971), 9-21 (1972). MR 47 \$31; II (Russian), ibid. 19 (1972), 87-96 (1973). MR $49 \$ 2980$.

7. Sylow p-subgroups of group algebras of countable abelian groups (Russian), C. R. Acad. Bulgare Sci. 27 (1974), 733-735. MR 51 \$5805.

8. D. S. Passman, Advances in group rings, Israel J. Math. 19 (1974), 67-107. MR $50 \$ 9945$.

Department of Mathematics, University of Arizona, Tucson, Arizona 85721 